\title{
Perforated duodenal ulcer: an unusual complication of gastroenteritis
}

\author{
Jane M Wilson, Christopher R Darby
}

\begin{abstract}
A 7 year old boy was admitted to hospital with gastroenteritis, which was complicated by an acute perforated duodenal ulcer. After oversewing of the perforation he made an uncomplicated recovery. Peptic ulceration is underdiagnosed in childhood and this leads to delay in diagnosis and appropriate management. Ulceration is associated with severe illness and viral infections, but perforation is rare.
\end{abstract}

As long ago as 1941 it was suggested that acute peptic ulceration in childhood was not rare, but that it was overlooked. Despite this, it does not feature prominently in the textbooks, where it is said to be an uncommon but well recognised complication of many serious diseases or severe stress in childhood. ${ }^{1}$ Children usually present with vomiting or gastrointestinal haemorrhage, or both, ${ }^{2}$ or the ulcer may be an accidental finding at necropsy. ${ }^{3}$ Perforation is rare. ${ }^{23} \mathrm{We}$ report perforation of an acute duodenal ulcer in a child who was not seriously ill but who was admitted with gastroenteritis.

\section{Case report}

A 7 year old retarded boy was admitted by his general practitioner with a 48 hour history of diarrhoea and vomiting. He had just spent the weekend at a children's respite home where most of the staff and residents had been struck down by similar symptoms. He was taking no drugs.

He had a history of birth asphyxia and delayed milestones, he had been unable to sit by the age of 8 months, and had generalised hypotonia at the age of 1 year. A translocation of chromosomes 1 and 4 was diagnosed, and this was thought to have contributed to his handicap. At the time of his presentation he was walking well, but was mentally retarded with considerable behavioural problems.

His maternal grandfather had had a duodenal ulcer diagnosed at endoscopy, and his maternal great grandfather was also said to have had an ulcer.

At presentation the boy was lethargic, miserable, and extremely dehydrated, though still passing urine. His abdomen was soft. Simple infective gastroenteritis was diagnosed, and he was admitted for oral rehydration.

Overnight the diarrhoea and vomiting subsided and he took oral fluids. By 18 hours after admission he was well hydrated and normal food was reintroduced. An hour later the vomit- ing recommenced and he had a large haematemesis and collapsed, becoming grey, cold, and clammy with unrecordable blood pressure and a heart rate of 170 beats/minute. His abdomen remained soft, but had become mildly distended. On rectal examination the faeces were soft and of normal colour.

After he had been resuscitated a chest radiograph was taken, which showed air under the diaphragm. At operation his abdomen was hugely distended, and a midline incision released a large amount of free gas; $1400 \mathrm{ml}$ of bile stained fluid contaminated with food were also released. The stomach and duodenum were exposed and a perforated acute duodenal ulcer $1 \mathrm{~cm}$ in diameter was found. After copious lavage the perforation and abdomen were closed and the patient made an uneventful recovery. Subsequent estimation of the serum gastrin concentration was normal.

\section{Discussion}

Peptic ulceration in children is not new: in 1826 a perforated gastric ulcer was reported in a 2 day old child. ${ }^{4}$ It has, however, been suggested that peptic ulceration is still underdiagnosed, and there are many uncertainties about its natural history. ${ }^{4}$

Peptic ulceration is rarely diagnosed in children, causing only between $3 \cdot 4 / 10000$, and 1/4700 admissions. ${ }^{5} 3$ At the Hospital for Sick Children, Toronto, only six perforations were detected in 20 years, ${ }^{3}$ at Montreal Children's Hospital eight in 11 years, ${ }^{2}$ and at the Children's Hospital, Pittsburgh, 11 in 20 years.

Childhood peptic ulcers are either 'primary' or 'secondary'. Primary ulcers present at a mean age of 8 years, the male:female ratio is $4: 1,^{3}$ there is often a strong family history, and they occur most commonly in patients with blood group 0.5 Secondary ulcers are precipitated by severe burns (Curling's ulcer), raised intracranial pressure (Cushing's ulcer), and other severe illnesses-for example, shock, sepsis, or cancer. In infants $80 \%$ of ulcers are secondary, but in older age groups the incidence drops to $20 \%{ }^{1}$

A recent study of 31 infants and children in whom acute duodenal ulceration had been diagnosed endoscopically showed that 29 (94\%) had a preceding illness: diarrhoea, respiratory infection, or fever. None of the ulcers had perforated. Evidence collected from that study suggested that acute duodenal ulceration can develop after viral illness, but heals quickly irrespective of the site and the number of ulcers. ${ }^{6}$ 
We thank Dr Martin Moncrieff for encouraging us to report this patient who was under his care, and for his helpful criticism of the manuscript.

1 Walker-Smith JA, Hamilton JR, Walker WA. Disorders of the stomach. Practical pediatric gastroenterology. London: Butterworth, 1983:174-84.

2 Decklebaum RJ, Roy CC, Lussier-Lazaroff J, Morin CL
Peptic ulcer disease: a clinical study in 73 children. Can Med Assoc F 1974;111:225-8.

3 Seagram CGF, Stephens CA, Cumming WA. Peptic ulceration at the Hospital for Sick Children, Toronto 1949-1969. $\mathcal{f}$ Pediatr Surg 1973;8:407-13.

4 Nord KS, Lebenthal E. Peptic ulcer in children: a review. Am $\mathcal{Y}$ Gastroenterol 1980;73:75-80.

5 Silverman A, Roy CC. Disorders of the stomach and duodenum. Pediatric clinical gastroenterology. St Louis: CV Mosby, 1983:162-89.

6 Hsu HY, Chang MH, Wang TH, et al. Acute duodenal ulcer. Arch Dis Child 1989;64:774-9.

\title{
An unusual cause of thoracic mass
}

\author{
D C Wilson, A O B Redmond
}

\begin{abstract}
A previously well 10 year old boy presented with scoliosis, a mass in the chest wall, and a pleural effusion. Chest radiography showed the triad of chronic consolidation, pleural effusion, and rib periostitis. Investigations confirmed thoracic actinomycosis. Tissue spread was evaluated by computed tomography. It was successfully treated with benzylpenicillin, which was later replaced by clindamycin.
\end{abstract}

Thoracic actinomycosis is extremely rare in children, especially if they are immunocompetent, and is not often considered in the differential diagnosis of paediatric pulmonary disease. This case illustrates the typical presentation, classic radiological and microbiological findings, and treatment of this subacute infection.

\section{Case report}

A 10 year old boy presented with lethargy, weight loss, and chest pain. A submandibular swelling and a mass in the chest wall had been noted recently. He had no history of anorexia, sweats, cough, fever, or sputum, but had mild asthma; he had not been in contact with tuberculosis.

He looked ill, but had no fever, lymphadenopathy, or rash. His weight was on the tenth centile. He had dental caries and diffuse swelling of the left submandibular area. There was a central abscess within a large, indurated, tender swelling of his left lower anterior chest wall. He had thoracic scoliosis, diminished expansion, dullness to percussion, and a patch of bronchial breathing with scattered crepitations at the left base. Liver and spleen were not enlarged.

Results of investigations included haemoglobin concentration $99 \mathrm{~g} / \mathrm{l}$, white cell count $16.3 \times 10^{9} / 1$, neutrophil count $13.7 \times 10^{9} / 1$, erythrocyte sedimentation rate $42 \mathrm{~mm}$ in the first hour, and $C$ reactive protein concentration 73 $\mathrm{mg} / \mathrm{l}$ (reference range $<6$ ). A chest radiograph showed thickening of the left chest wall, consolidation of the left lower lobe, left pleural effusion, and periostitis of the left seventh and

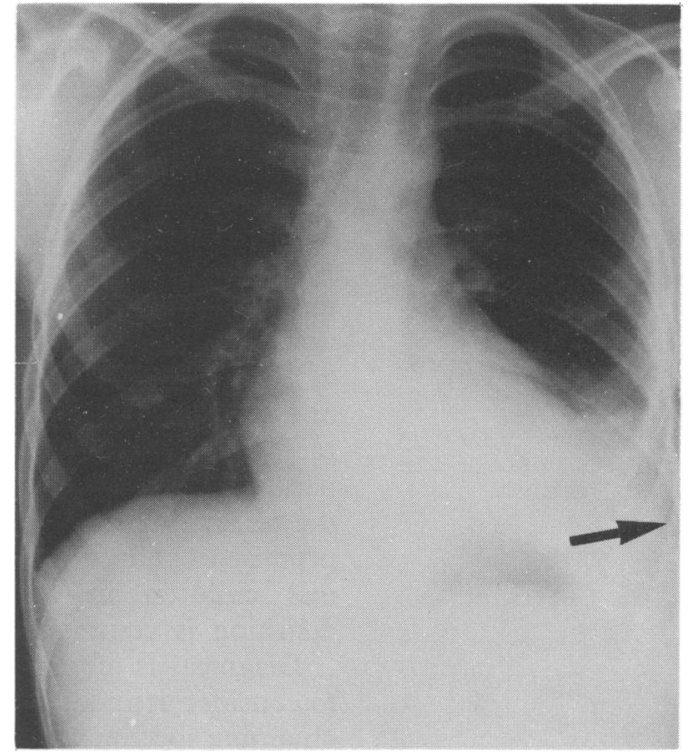

Chest radiograph showing triad of chronic consolidation, pleural effusion, and rib periostitis (arrowed).

eighth ribs (figure). A computed tomogram of the thorax showed a lesion spreading from the left lower lobe of the lung to the pleura, invading the ribs, and then passing through the chest wall. Radiographs of the mandible, abdominal ultrasound scan, liver function tests, and immunological screen were normal. Blood cultures grew no pathogens and a Mantoux test was negative.

When the abscess pointed, it was incised. Examination of the pus showed sulphur granules and Gram positive branching filaments with clubs; Actinomyces israelii was grown. High doses of benzylpenicillin were given intravenously, and four carious teeth extracted. After two weeks the erythrocyte sedimentation rate was $14 \mathrm{~mm} /$ in the first hour and $C$ reactive protein concentration $7 \mathrm{mg} / \mathrm{l}$. One week later, as he had developed urticaria, clindamycin was substituted for benzylpenicillin. After five weeks of parenteral treatment, he was discharged taking clindamycin orally. This was continued for eight months and during this time his weight increased and the chest signs resolved. A
Accepted 5 April 1990

(Arch Dis Child 1990;65:991-2) 